\title{
Food Safety Risk Communication between the Food Regulator and Consumer in China: An Evolutionary Game Perspective
}

\author{
Ying Zhu $\mathbb{D},{ }^{1}$ May Chu $\mathbb{D},{ }^{2}$ Xiaowei Wen $\mathbb{D},,^{1,3}$ and Yiqin Wang $\mathbb{D}^{1}$ \\ ${ }^{1}$ College of Economics \& Management, South China Agricultural University, Guangzhou 510642, China \\ ${ }^{2}$ Department of Government and Public Administration, Chinese University of Hong Kong, Hong Kong 999077, China \\ ${ }^{3}$ Research Institute of Rural Development of Guangdong Province, Guangzhou 510642, China
}

Correspondence should be addressed to Xiaowei Wen; wxwcn@126.com

Received 11 March 2021; Accepted 6 August 2021; Published 18 August 2021

Academic Editor: Fanlin Meng

Copyright ( $\odot 2021$ Ying Zhu et al. This is an open access article distributed under the Creative Commons Attribution License, which permits unrestricted use, distribution, and reproduction in any medium, provided the original work is properly cited.

Food safety risk communication has attracted widespread attention in China. The government is the leader in food risk communication. It has even more impact on consumers' willingness to communicate food risk. Thus, this paper constructs a risk communication game model composed of the central government, local government, and consumers under food safety regulatory agencies in China. Based on the evolutionary game theory, we achieved the evolutionary stable equilibrium points under complying different constraint conditions by solving the replicator dynamic equations of parties in the dynamic system. In the end, some numerical examples have been displayed to simulate the agents' choices. The results show that penalty cost, governance intensity, communication cost, and reputation of the government have an essential impact on consumers' enthusiasm for food safety risk communication decision-making behavior. Furthermore, the choice of food safety risk communication strategies is performed with a dynamic process, which is constantly adjusted and optimized. The evolutionary game system can converge on an ideal state under certain conditions. Moreover, this study proposes suggestions and measures conducive to the tripartite game, which can offer valuable guidance on food risk communication practice and improve government efficiency in China. This research can provide the evolutionary mechanism and broaden our understanding of the relationship between food safety risk governance and food risk communication strategies.

\section{Introduction}

The field of research into risk communication has recently become broad and diverse. Risk communication became recognized as a necessary component in risk management [1]. The US National Research Council Committee defines risk communication as an interactive process of exchanging information and opinion among individuals, groups, and institutions. It involves multiple messages, not strictly about risk, which express concerns, opinions, or reactions to risk messages or legal and institutional arrangements for risk management [2].

According to the European Food Safety Authority (EFSA) [3], the goal of risk communication is to give stakeholders an understanding of the basic principles of risk decision-making so that they can make an informed judgment. Communications in a food safety crisis should be timely, reliable, transparent, including all stakeholders and adopt appropriate channels to convey risk information [4].

After defining and understanding risk communication, the question "how is risk in processed food or other items communicate to consumers" can be answered. Reasonably significant is the development and enacting of food safety policies and regulation of food safety legislation by individual countries [5]. Food regulation is an integral part of public health, and it affects the public health and the healthy development of the food industry. Specifically, it reduces morbidity and mortality associated with consuming contaminated food [6]. In addition to regulators, distinguishing the attitudes of consumers or identifying their sociodemographic characteristics are crucial for reducing 
consumers' exposure to food risk [7]. Food safety regulator is a vital component of food safety risk communication. A core risk communication strategy for food safety regulators was developed by Charlebois and Summan [8]. The strategy contributes to minimizing food risk effects by influencing stakeholder behavior or improving interactions between the food regulatory and relevant stakeholders. Regulators could implement effective risk management by designing effective communication measures on food safety hazards, thereby reducing the serious consequences caused by food risks [9]. The prevention and control of food safety risk communication have been paid more attention, so that the government takes comprehensive responses strategy and appropriate action to improve communication effectiveness. Boholm designed a practice-based approach to study the government agency officials' experience of what characterizes successful and failed risk communication [10]. Most previous studies have explored the prevention and control measures of food safety risk communication from the perspective of government or regulators $[8,11,12]$. In this regard, previous researches have focused on exploring how supervisors can communicate food safety information to consumers effectively. Food regulatory agencies make efforts to protect public health and regulate the market order of the food industry to reduce foodborne illness or food-related mortality.

However, there are many challenges associated with food regulation. For example, there is no food safety risk communication system with consumers in China, and food safety issues are complex to identify. Furthermore, there is no interactive public platform for food safety risk communication and a database of food safety issues across the country for consumers to facilitate access and inquiry. Thus, the rest of this study is organized as follows. We present the literature review in Section 2. Section 3 constructs the evolutionary game model of food risk communication stakeholders. Section 4 discusses the evolutionary stability of the game model. Section 5 reports the numerical simulation results. Section 6 describes the conclusions and contributions of this study.

\section{Literature Review}

2.1. Food Safety Risk Communication in China. Food Safety Law was implemented in 2009 and amended in 2018. Food safety risk communication systems have been formulated, which marks the legalization of food safety risk communication in China. Article 23 of Food Safety Law clearly states: "the food safety regulatory agency and management department of the people's government of the county or above level, the risk assessment expert committee and technical institutions shall follow the principles of science, objectivity, timeliness, and openness to organize food producers and operators, food inspection agencies, certification agencies, food industry associations, consumer associations, and news media to communicate assessment information and supervision information." It seems that the government is expecting more public engagement in food safety supervision.
Actually, the central government coordinates and leads the Food Safety Commission of the State Council in charge of food safety supervision and management throughout the country. The Ministry of Agriculture and Rural Affairs, State Administration for Market Regulation, and the National Health Commission constitute the food safety supervision and management agency (see Figure 1). The Chinese Food and Drug Administration (CDFA) and the National Health and Family Planning Commission of China (NHFPC) have crucial responsibilities in implementing food safety regulation [15]. The establishment of these two institutions marks that food safety governance has entered a legal track. Specifically, the local governments have direct contact with consumers to communicate on food safety risks. They release food safety information through the local market supervision and administration bureau and deliver the market food random inspections to consumers, but this is one-way risk communication. Generally, the CFSA (China National Center for Food Safety Risk Assessment), a public health institution approved by the Office of the Central Organization Committee, is the national technical agency responsible for food safety risk assessment, provides technical support for national food safety risk assessment, monitoring, early warning, communication, and food safety standards.

Therefore, food safety risk communication is led by the government, and government-led food safety exchange information is often transmitted in scientific language that is difficult for the public. At the same time, the Chinese government has been making great efforts in legislation and institution building. The government has continuously released and suppressed social forces to compensate for the weak regulatory capabilities [16]. In addition to the establishment of the food safety system, the specific food safety standard formulation process requires greater transparency and strictness than in the past [17]. May focused on food safety supervision in China and pointed out the relatively weak state regulatory capacity [18]. Some scholars have also focused on web-based tools specifically designed for young people as a food risk communication tool [19]. Currently, it is necessary for demand with two-way communication on food safety risks between consumers and regulators urgently in China. Most people think "risk" is terrible news in China. Almost all people will be resistant to communicate food risk when they listen to the term of risk. Not only do the public not understand the content, subject, concept, strategy, and form of food safety risk communication, but even professional practitioners in the food safety field have a one-side understanding of food safety risk communication.

In previous studies, there are substantial differences in how to rank the importance of various food hazards between the experts and laypersons for various reasons [20-22]. In other words, experts and consumers have differences in comprehensive ability and risk perception on food. However, the consumers also felt that their knowledge of food risk was more than other people, giving rise to an "illusion of knowledge" [23]. For food regulatory agencies, it is necessary to understand the public's views on food safety risks and formulate effective risk communication measures [24]. The effectiveness of a communication strategy depends on the 


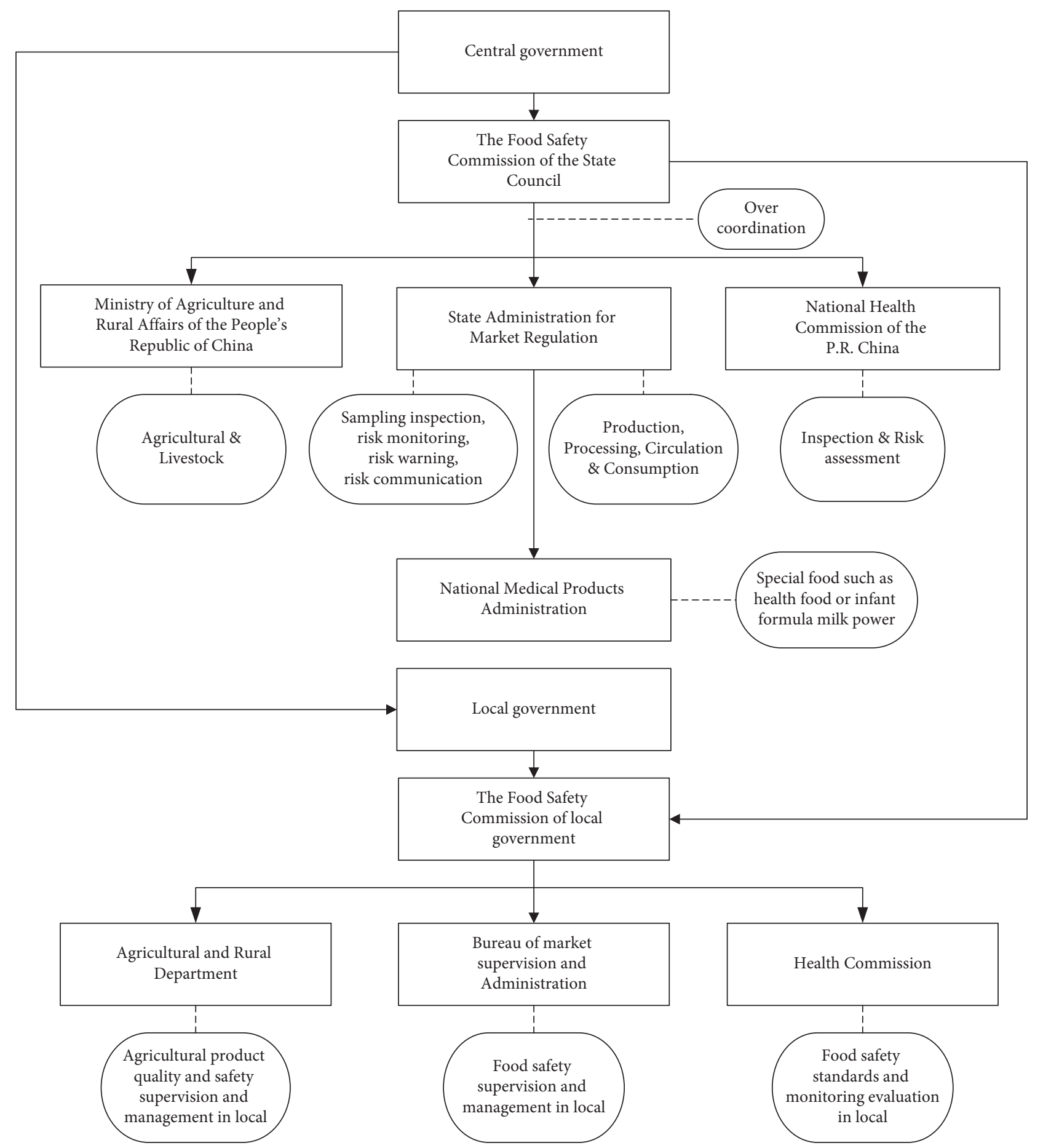

\footnotetext{
Department
}

Resposibility

FIgURE 1: The current government authorities involved in food safety governance in China. Source: adapted from [13, 14].

critical element that the audience cared most about [25]. Considering the situation surrounding food safety risk communication in China, it is hard to say that the concept of risk communication has been accurately appreciated and established in public. Consumers are engaged in food risk communication and achieved message effectively have not gained considerable scholarly attention in China.
2.2. Evolutionary Game Theory in Food Safety Governance. The evolutionary game theory has been widely used in the research of food safety governance. A plethora of studies have examined the governance strategy on food safety with the game model. For example, Song et al. established the evolutionary game model to analyze food safety information disclosure between food enterprises and 
government regulators [26]. Through simulation experiments, they found that false food safety information cannot be solved effectively, only relying on government supervision. Scholars construct food safety evolutionary game model from different theoretical perspectives [27-29]. The results showed that it is difficult to achieve the stable evolutionary strategy with the constraint conditions [27]. Further, Yang et al. designed an evolutionary game model to study evolutionarily stable strategies of food suppliers and producers. Their results showed that the behavioral strategies are the hitchhiking benefits, the cost of quality-improving efforts, and the initial strategy [30]. According to Neuwirth et al., the response efficacy of consumers is positively correlated with communicating intentions and self-efficacy, but it demonstrates a negative relationship to perceived barriers [31]. Luo et al. developed a three-party game model of health food safety risk evolution, which includes health food enterprises, consumers, and government regulators. They conclude that the influence mechanism of factors such as information search payment, consumers' subjective perception, and regulatory authority's certification affect food safety risks [28]. Based on the above, Luo et al. argue that consumers' sensitivity to health food safety supervision information can help improve the profitability of enterprises [29].

Food risk communication must take account of the actual concerns of the public [32]. In food risk communication, the public pays more attention to the timeliness, transparency of information, perceived risks, and controllability of risks during a crisis [33]. Specifically, the public's perception of risk significantly affects their information needs [34]. Currently, regulators and some academics call on the public and stakeholders to participate frequently in the decision-making process [35]. Effective communication barriers include personal, infrastructure, and message-related factors [36]. To provide relevant risk information to the target group requires an in-depth understanding of the characteristics of the audience, such as age, knowledge level, consumption habits, etc. [37]. Traditional risk communication is difficult to succeed independently. More innovative communication strategies are needed to interact in an open way for the target group [38].

However, the above studies ignored the constant adjustment of the regulatory behavior of regulators and the communication behavior of consumers. To change the status of consumers passively receiving food information, how to achieve a stable equilibrium between consumer communication behavior and regulators' regulatory behavior needs to be focused on. Therefore, this study aims to explore the risk communication behavior of stakeholders and identifies influence factors that contribute to the success and failure of food safety risk communication work practice from the regulators in China. Applying the evolutionary game theory in the food safety risk communication enriches the food risk theory. This paper analyzes the replication of dynamic games under bounded rational conditions, and the agents need continuous dynamic adjustment behavior to achieve a tripartite evolutionary stability strategy. The contribution of the research is in the presentation of food safety risk communication systems and strategies used by consumers to improve communication of food risks.

\section{Evolutionary Game Modeling for Food Safety Risk Communication Behavior}

3.1. Basic Research Assumptions and Strategic Choices under Food Governance Mechanism in China. The evolutionary game theory model is an effective method to investigate the strategy interaction process among game agents in the fields of wireless communications and networking, Network security, etc. Zhang and Guizani used the game theory model to explore the decision-making problem in wireless communications and networking and showed that the networks converge basically into noncooperative and cooperative states [39]. Zhu and Başar focused on the competitive and strategic behaviors between an attacker and a defender with a game model, which aims to develop defense solutions to deter and mitigate the attacks [40]. Boholm described interorganizational collaboration and assigning of responsibility contributing to risk communication [10]. Annabelle et al. reported consumers to communicate with food regulators, including reactive and proactive communication in two states [12].

The above research results provide a good reference for us. In this part, we formulated a system model between regulators and consumers, which is shown in Figure 2. Thus, for the convenience of analysis, the relevant assumptions and parameters are stipulated as follows:

Assumption 1. Food safety risk communication including local government, consumers, and central government. The strategy space of local government is to perform food safety risk communication duties. Consumers' strategy space is proactive communication and reactive communication. The strategy space of central government is strong supervision and weak supervision.

Assumption 2. Supposing that the probability of local government choosing to perform food safety risk communication duties is $\xi \in[0,1]$; the probability of choosing not to perform food safety risk communication duties is $1-\xi$; the probability of consumer choosing proactive communication is $\gamma \in[0,1]$; the probability of choosing reactive communication is $1-\gamma$; the probability of central government choosing strong supervision is $\eta \in[0,1]$; the probability of choosing weak supervision is $1-\eta$.

Assumption 3. $D_{11}$ is the return of the local government. $C_{11}$ is the cost of local government performing food safety risk duties; $C_{12}$ is the cost of local government not performing food safety risk duties. $R$ is the reward of performing food safety risk communication duties from the central government with strong supervision when consumers choose proactive communication. $\pi$ is the possibility of being discovered when local government fails to perform food safety risk communication duties under weak supervision. $k$ is the central government's penalties for local government failing to perform food safety risk communication duties 


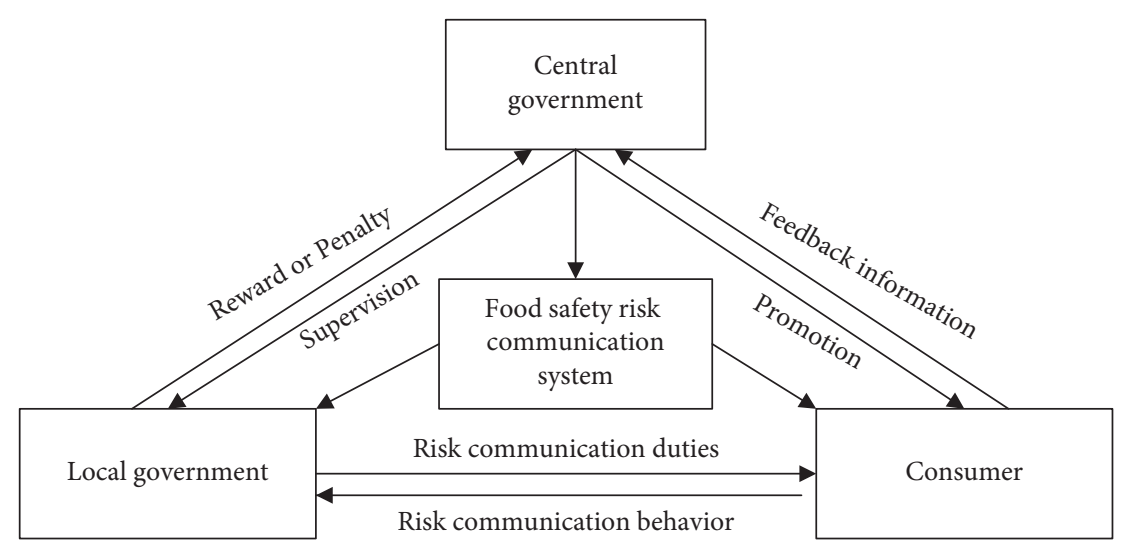

Figure 2: System model between regulators and consumers.

during strong supervision. $S$ is the extra return of local government when it does not perform food safety risk communication duties. $L_{11}$ is the negative effect on local government reputation when it does not perform food safety risk communication duties when the consumer chooses proactive communication. $F$ is the potential loss for local government when it performs food safety risk communication duties while consumers choose reactive communication. $B_{11}$ is credibility enhancement of local government due to performing risk communication duties.

Assumption 4. $D_{21}$ is the return of consumer choosing proactive communication when the local government performs food safety risk communication duties. $D_{22}$ is shortterm gains from consumers' reactive communication during local government performing food safety risk communication duties. $D_{23}$ is the return from consumers' proactive communication during local government not performing food safety risk communication duties. $C_{21}$ is the cost for consumers choosing proactive communication to learn more information about food safety issues. $C_{22}$ is the cost for consumers to choose reactive communication. $L_{21}$ is the potential loss of consumers' reactive communication in food safety issues under the local government performing food safety risk communication duties. $B_{21}$ is a positive effect on consumers' proactive communication under strong supervision. $B_{22}$ is the extra return for a consumer choosing proactive communication when local government performs food safety risk communication duties under strong supervision.

Assumption 5. $D_{31}$ is the revenue of the central government. $C_{31}$ is the cost for the central government to implement strong supervision. $C_{32}$ is the cost for central government to implement weak supervision. $B_{31}$ is the credibility enhancement of central government when local government performs food safety risk communication duties under strong supervision. $L_{31}$ is the loss of credit of central government due to consumer proactive communication and local government failure to perform food safety risk communication duties. $W$ is social welfare improvement with proactive communication of consumers in a long time. All parameters are greater than zero.

Summarizing above, the model parameters used in this paper can be listed in Table 1 .

3.2. Payoff Matrix of the Tripartite Game Model. In the case of the regulator having obligations to communicate with the consumer on food safety risks according to the Food Safety Law of China, both players incur different costs depending on the strategies adopted. In the vertical hierarchical management of the Chinese government, we divided regulators into two types: central government and local government. Regarding the above assumptions, the payoff matrix of players is presented in Table 2 .

\section{Evolutionary Stability Analysis}

4.1. Replicator Dynamic Equations of Players. According to the evolutionary game theory, we let the expected return of local government performing risk communication duties strategy be $E_{1 L}$, and let the expected return of local government under the not performing risk communication duties strategy be $E_{2 L}$. Then, we can obtain the excepted return of local government that performs and does not perform food risk communication duties as follows:

$$
\begin{aligned}
E_{1 L}= & \gamma \eta\left(D_{11}-C_{11}+R+B_{11}\right)+(1-\gamma) \eta\left(D_{11}-C_{11}+B_{11}-F\right) \\
& +\gamma(1-\eta)\left(D_{11}-C_{11}+B_{11}\right)+(1-\gamma)(1-\eta)\left(D_{11}-C_{11}-F\right), \\
E_{2 L}= & \gamma \eta\left(D_{11}-C_{12}-L_{11}-k S\right)+(1-\gamma) \eta\left(D_{11}-C_{12}-k S\right) \\
& +\gamma(1-\eta)\left(D_{11}-C_{12}-L_{11}-\pi k S\right)+(1-\gamma)(1-\eta)\left(D_{11}-C_{12}-\pi k S\right) .
\end{aligned}
$$


TABLE 1: Description of model parameters.

\begin{tabular}{|c|c|}
\hline Variables & Implications \\
\hline$\overline{D_{11}}$ & The returns of local government on the food risk communication duties \\
\hline$C_{11}$ & Cost for local government to perform risk communication duties \\
\hline$C_{12}$ & Cost for local government not to perform risk communication duties \\
\hline$R$ & $\begin{array}{c}\text { Reward of local government performing food safety risk communication duties from central government with strong } \\
\text { supervision when the consumer chooses proactive communication }\end{array}$ \\
\hline$\pi$ & The possibility of local government not performing risk communication duties will be discovered under weak supervision \\
\hline$k$ & $\begin{array}{c}\text { Degree of punishment from the central government after finding that local government does not perform risk communication } \\
\text { duties during strong supervision }\end{array}$ \\
\hline$S$ & Extra return if local governr \\
\hline$L_{11}$ & $\begin{array}{c}\text { Negative effect on the reputation of local government not performing risk communication duties when the consumer chooses } \\
\text { proactive communication }\end{array}$ \\
\hline$F$ & $\begin{array}{l}\text { Potential loss for local government with performance risk communication duties if the consumer chooses reactive } \\
\text { communication }\end{array}$ \\
\hline$B_{11}$ & Credibility enhancement of local governm \\
\hline$\xi$ & The probability that local government \\
\hline$D_{21}$ & The return of consumer choosing proactive con \\
\hline$D_{22}$ & Short-term gains of consumer due to reactive communication when the local government performs risk communication duties \\
\hline$D_{23}$ & $\begin{array}{l}\text { The return of the consumer choosing proactive communication if a local government chooses not to perfor } \\
\text { communication duties }\end{array}$ \\
\hline$C_{21}$ & $\begin{array}{r}\text { Cost for the consumer choosing proactive communication to learn mor } \\
\text { economy, and opport }\end{array}$ \\
\hline$C_{22}$ & Cost for the consumer choos \\
\hline$L_{21}$ & $\begin{array}{l}\text { Potential loss of consumer's reactive communication in food safety issues under the local government performs risk } \\
\text { communication duties }\end{array}$ \\
\hline$B_{21}$ & Positive effect on consumer choosing proactive com \\
\hline$B_{22}$ & $\begin{array}{c}\text { Extra return for the consumer when choosing proactive communication if local government performs risk communication } \\
\text { duties under strong supervision }\end{array}$ \\
\hline$\gamma$ & The probability that the cons \\
\hline$D_{31}$ & The revenu \\
\hline$C_{31}$ & Cost for central government to implement strong supe \\
\hline$C_{32}$ & Cost for central government \\
\hline$B_{31}$ & $\begin{array}{c}\text { Credibility enhancement of central government due to local government perform risk communication duties under strong } \\
\text { supervision }\end{array}$ \\
\hline$L_{31}$ & $\begin{array}{c}\text { Credibility loss of the central government under proactive communication due to local government does not perform risk } \\
\text { communication duties }\end{array}$ \\
\hline$W$ & Social welfare improvement with proactive communication of food safety risks by the consumer \\
\hline$\eta$ & The probability that the central government chooses strong supervision \\
\hline
\end{tabular}

TABle 2: Payoff matrix of the tripartite game.

\begin{tabular}{|c|c|c|c|}
\hline \multirow{2}{*}{ Local government } & \multirow{2}{*}{ Consumer } & \multicolumn{2}{|c|}{ Central government } \\
\hline & & Strong supervision $(\eta)$ & Weak supervision $(1-\eta)$ \\
\hline Performing risk communication duties $(\xi)$ & $\begin{array}{c}\text { Proactive communication }(\gamma) \\
\text { Reactive communication }(1-\gamma)\end{array}$ & $\begin{array}{c}D_{11}-C_{11}+R+B_{11} \\
D_{21}-C_{21}+B_{21}+B_{22} \\
D_{31}-C_{31}+B_{31}+W \\
D_{11}-C_{11}+B_{11}-F \\
D_{22}-C_{22}-L_{21} \\
D_{31}-C_{31}+B_{31} \\
\end{array}$ & $\begin{array}{c}D_{11}-C_{11}+B_{11} \\
D_{21}-C_{21} \\
D_{31}-C_{32} \\
D_{11}-C_{11}-F \\
D_{22}-C_{22}-L_{21} \\
D_{31}-C_{32} \\
\end{array}$ \\
\hline Not performing risk communication duties $(1-\xi)$ & Reactive communication $(1-\gamma)$ & $\begin{array}{c}D_{11}-C_{12}-L_{11}-k S \\
D_{23}-C_{21}+B_{21} \\
D_{31}-C_{31}+k S \\
D_{11}-C_{12}-k S \\
0 \\
D_{31}-C_{31}+k S\end{array}$ & $\begin{array}{c}D_{11}-C_{12}-L_{11}-\pi k S \\
D_{23}-C_{21} \\
D_{31}-C_{32}-L_{31} \\
D_{11}-C_{12}-\pi k S \\
0 \\
D_{31}-C_{32} \\
\end{array}$ \\
\hline
\end{tabular}

The average expected return is as follows:

$$
\bar{E}_{L}=\xi E_{1 L}+(1-\xi) E_{2 L} .
$$

According to the dynamic equation of Malthusian, the replicator dynamics equation of local government's strategy is as follows: 


$$
\begin{aligned}
& \frac{\mathrm{d} \xi}{\mathrm{d} t}=\xi\left(E_{1 L}-\bar{E}_{L}\right)=\xi(1-\xi)\left(E_{1 L}-E_{2 L}\right) \\
& =\xi(1-\xi)\left[\gamma \eta\left(R-B_{11}\right)+\eta\left(B_{11}+k S-\pi k S\right)+\gamma\left(B_{11}+L_{11}+F\right)+\pi k S+C_{12}-C_{11}-F\right] . \\
& E_{1 G}=\xi \gamma\left(D_{31}-C_{31}+B_{31}+W\right)+\xi(1-\gamma)\left(D_{31}-C_{31}+B_{31}\right) \\
& +(1-\xi) \gamma\left(D_{31}-C_{31}+k S\right)+(1-\xi)(1-\gamma)\left(D_{31}-C_{31}+k S\right), \\
& E_{2 G}=\xi \gamma\left(D_{31}-C_{32}\right)+\xi(1-\gamma)\left(D_{31}-C_{32}\right) \\
& +(1-\xi) \gamma\left(D_{31}-C_{32}-L_{31}\right)+(1-\xi)(1-\gamma)\left(D_{31}-C_{32}\right) .
\end{aligned}
$$

Let $E_{1 C}$ represent the expected return of consumers if they choose proactive communication and $E_{2 C}$ represent the expected return of consumers if they choose reactive communication. Then, we can obtain the excepted return of consumer that proactive and reactive risk communication on food is as follows:

$$
\begin{aligned}
E_{1 C}= & \xi \eta\left(D_{21}-C_{21}+B_{21}+B_{22}\right)+\xi(1-\eta)\left(D_{21}-C_{21}\right) \\
& +(1-\xi) \eta\left(D_{23}-C_{21}+B_{21}\right)+(1-\xi)(1-\eta)\left(D_{23}-C_{21}\right), \\
E_{2 C}= & \xi \eta\left(D_{22}-C_{22}-L_{21}\right)+\xi(1-\eta)\left(D_{22}-C_{22}-L_{21}\right) \\
& +(1-\xi) \eta \times 0+(1-\xi)(1-\eta) \times 0 .
\end{aligned}
$$

The average expected return of consumers can be written as follows:

$$
\bar{E}_{C}=\gamma E_{1 C}+(1-\gamma) E_{2 C} .
$$

The replicator dynamics equation of consumer's strategy can be expressed as follows:

$$
\begin{aligned}
\frac{\mathrm{d} \gamma}{\mathrm{d} t}= & \gamma\left(E_{1 C}-\bar{E}_{C}\right)=\gamma(1-\gamma)\left(E_{1 C}-E_{2 C}\right) \\
= & \gamma(1-\gamma)\left[\xi\left(D_{21}-D_{22}-D_{23}+C_{22}+L_{21}\right)\right. \\
& \left.+\xi \eta B_{22}+\eta B_{21}+D_{23}-C_{21}\right] .
\end{aligned}
$$

Let $E_{1 G}$ represent the expected return of central government if they choose strong supervision and $E_{2 G}$ represent the expected return of central government if they choose weak supervision. Then, we can obtain the excepted return of central government that strong and weak supervision is as follows:
The average expected return of central government can be written as follows:

$$
\bar{E}_{G}=\eta E_{1 G}+(1-\eta) E_{2 G} .
$$

The replicator dynamics equation of central government can be expressed as follows:

$$
\begin{aligned}
\frac{\mathrm{d} \eta}{\mathrm{d} t}= & \eta\left(E_{1 G}-\bar{E}_{G}\right)=\eta(1-\eta)\left(E_{1 G}-E_{2 G}\right) \\
= & \eta(1-\eta)\left[\xi \gamma\left(W-L_{31}\right)+\xi\left(B_{31}-k S\right)+\gamma L_{31}\right. \\
& \left.+k S+C_{32}-C_{31}\right] .
\end{aligned}
$$

The replicator dynamic equations (3), (6), and (9) constitute a three-dimensional dynamic system as follows:

$$
\left\{\begin{array}{l}
F(\xi)=\frac{\mathrm{d} \xi}{\mathrm{d} t}=\xi(1-\xi)\left(E_{1 L}-E_{2 L}\right), \\
F(\gamma)=\frac{\mathrm{d} \gamma}{\mathrm{d} t}=\gamma(1-\gamma)\left(E_{1 C}-E_{2 C}\right), \\
F(\eta)=\frac{\mathrm{d} \eta}{\mathrm{d} t}=\eta(1-\eta)\left(E_{1 G}-E_{2 G}\right),
\end{array}\right.
$$

where the concrete forms of $E_{1 L}-E_{2 L}, E_{1 C}-E_{2 C}$, and $E_{1 G}-$ $E_{2 G}$ can be expressed as follows:

$$
\left\{\begin{array}{l}
E_{1 L}-E_{2 L}=\gamma \eta\left(R-B_{11}\right)+\eta\left(B_{11}+k S-\pi k S\right)+\gamma\left(B_{11}+L_{11}+F\right)+\pi k S+C_{12}-C_{11}-F, \\
E_{1 C}-E_{2 C}=\xi\left(D_{21}-D_{22}-D_{23}+C_{22}+L_{21}\right)+\xi \eta B_{22}+\eta B_{21}+D_{23}-C_{21}, \\
E_{1 G}-E_{2 G}=\xi \gamma\left(W-L_{31}\right)+\xi\left(B_{31}-k S\right)+\gamma L_{31}+k S+C_{32}-C_{31} .
\end{array}\right.
$$

4.2. Evolutionary Stability Strategy of Players. According to the replicator dynamic equations of the model, the probability of performing risk communication duties by local government $\xi(t)$, the probability of proactive communication by a consumer $\gamma(t)$, and the probability of strong supervision by the central government $\eta(t)$ all depend on time $t$, and $\xi(t), \gamma(t), \eta(t) \in[0,1]$. In the system, we let $F(\xi)=0$, $F(\gamma)=0, F(\eta)=0$, the dynamical system has eight local equilibrium points of adopting pure strategies as follow: $(0$, $0,0),(0,0,1),(0,1,0),(1,0,0),(1,1,0),(1,0,1),(0,1,1)$, and $(1,1,1)$.
4.2.1. The Evolutionarily Stable Strategy of the Local Government. In formula (3), if $E_{1 L}-E_{2 L}=0$, the solution of the equation is $\gamma=\gamma^{*}=((F+\eta \pi k S-\eta k S$ $\left.\left.-\pi k S-\eta B_{11}+C_{11}-C_{12}\right) /\left(\eta R-\eta B_{11}+B_{11}+L_{11}+F\right)\right)$, then the dynamic equation satisfies $F(\xi) \equiv 0$, that is to say, when the probability of proactive communication by consumer and the probability of strong supervision satisfy these above conditions, the local government will always choose not to perform risk communication duties strategy, which would be a stable strategy for local government, as demonstrated in Figure 3. Then, the evolutionary stable strategies 


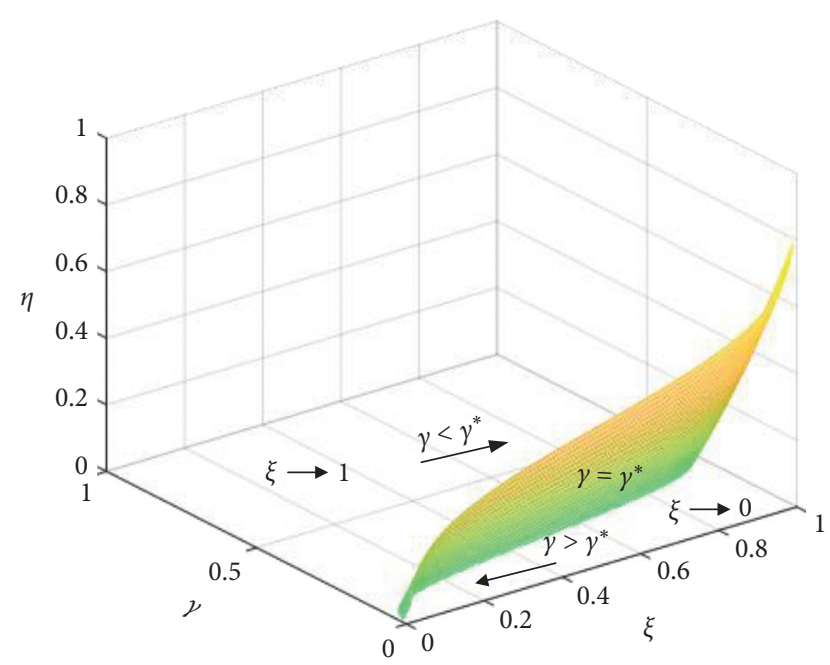

FIgURE 3: Evolutionary phase diagram of local government's strategy selection.

of performing risk communication responsibility behavior of local government are obtained as follows:

If $E_{1 L}-E_{2 L} \neq 0$, let $F(\xi)=0$, then $\xi=0$ and $\xi=1$ are stable points. By solving the partial derivative of replicator dynamics equation (3), we could obtain the following:

$$
\frac{\mathrm{d} F(\xi)}{\mathrm{d} \xi}=(1-2 \xi)\left(E_{1 L}-E_{2 L}\right)
$$

Obviously, $\eta R-\eta B_{11}+B_{11}+L_{11}+F>0$, we consider the following scenarios:

Scenario 1: according to equation (12), if $E_{1 L}-E_{2 L}<0$, that is $\quad \gamma>\gamma^{*}$, then $\left.(\mathrm{d} F(\xi) / \mathrm{d} \xi)\right|_{\xi=0}<0$, $\left.(\mathrm{d} F(\xi) / \mathrm{d} \xi)\right|_{\xi=1}>0$. Thus $\xi=0$ is the evolutionary stable point. It means the unique evolutionary stable strategy under the above constraint conditions. It is showing that local government tends to choose not to perform risk communication duties as their stable strategy. When the probability of proactive communication by the consumer is large enough, local government will choose not to perform food safety risk communication duties, and not performing risk communication duties would be their optimal strategy.

Scenario 2: if $E_{1 L}-E_{2 L}>0$, that is $\gamma<\gamma^{*}$, then $\left.(\mathrm{d} F(\xi) / \mathrm{d} \xi)\right|_{\xi=0}>0,\left.(\mathrm{~d} F(\xi) / \mathrm{d} \xi)\right|_{\xi=1}<0$. Thus $\xi=1$ is the evolutionary stable point, representing the unique evolutionary stable strategy. It indicates that local government will choose to perform risk communication duties as their stable strategy. When the probability of proactive communication by the consumer is small enough, the local government tends to perform food safety risk communication duties, and perform risk communication duties would be their optimal strategy.

4.2.2. The Evolutionarily Stable Strategy of the Consumer. In the formula (6), if $E_{1 C}-E_{2 C}=0$, the solution of the equation is $\eta=\eta^{*}=\left(\left(\xi D_{22}+\xi D_{23}-\xi \quad D_{21}-\xi C_{22}-\right.\right.$ $\left.\left.\xi L_{21}+C_{21}-D_{23}\right) /\left(B_{21}+\xi B_{22}\right)\right)$, then the dynamic equation satisfies $F(\gamma) \equiv 0$, which indicate that when the probability of performing risk communication duties by local government and the probability of strong supervision satisfy these above conditions, the consumer will always adopt the reactive communication strategy, which would be a stable strategy for consumer, as shown in Figure 4.

If $E_{1 C}-E_{2 C} \neq 0$, let $F(\gamma)=0$, then $\gamma=0$ and $\gamma=1$ are stable points. Next, we need to solve the partial derivative of replicator dynamics equation (6), then we could obtain the following:

$$
\frac{\mathrm{d} F(\gamma)}{\mathrm{d} \gamma}=(1-2 \gamma)\left(E_{1 C}-E_{2 C}\right)
$$

Obviously, $B_{21}+\xi B_{22}>0$, we consider the following scenarios:

Scenario 1: according to equation (13), if $E_{1 C}-E_{2 C}<0$, that is $\eta<\eta^{*}$, then $\left.(\mathrm{d} F(\gamma) / \mathrm{d} \gamma)\right|_{\gamma=0}<0$, $\left.(\mathrm{d} F(\gamma) / \mathrm{d} \gamma)\right|_{\gamma=1}>0$. Thus, $\gamma=0$ is an evolutionary stable point, which means that this stable point is the globally unique evolutionary stable strategy. It is showing that consumers tend to choose reactive communication as their stable strategy. When the probability of strong supervision by the central government is small enough, the consumer tends to reactive communication, and reactive communication would be their optimal strategy.

Scenario 2: if $E_{1 C}-E_{2 C}>0$, that is, $\eta>\eta^{*}$, then $\left.(\mathrm{d} F(\gamma) / \mathrm{d} \gamma)\right|_{\gamma=0}>0,\left.(\mathrm{~d} F(\gamma) / \mathrm{d} \gamma)\right|_{\gamma=1}<0$. Thus $\gamma=1$ is the evolutionary stable point. It means that the sable point is the globally unique evolutionary stable strategy. It indicates that the consumer tends to choose proactive communication as their stable strategy. When the probability of strong supervision by the central government is large enough, the consumer tends to choose proactive communication, and proactive communication would be their optimal strategy.

4.2.3. The Evolutionarily Stable Strategy of the Central Government. In formula (9), if $E_{1 G}-E_{2 G}=0$, the solution of the equation is $\xi=\xi^{*}=\left(\left(C_{31}-C_{32}-\gamma L_{31}\right.\right.$ $\left.-k S) /\left(\gamma W+B_{31}-\gamma L_{31}-k S\right)\right)$, then the dynamic equation satisfies $F(\eta) \equiv 0$, which indicates that when the probability of performing risk communication duties by local government and the probability of proactive communication by consumers satisfy these above conditions, the central government will always choose weak supervision strategy, which would be a stable strategy for central government, as shown in Figure 5.

If $E_{1 G}-E_{2 G} \neq 0$, let $F(\eta)=0$, then $\eta=0$ and $\eta=1$ are stable points. We solve the partial derivative of replicator dynamics equation (9); we could obtain the following:

$$
\frac{\mathrm{d} F(\eta)}{\mathrm{d} \eta}=(1-2 \eta)\left(E_{1 G}-E_{2 G}\right) .
$$

We consider the following scenarios: 


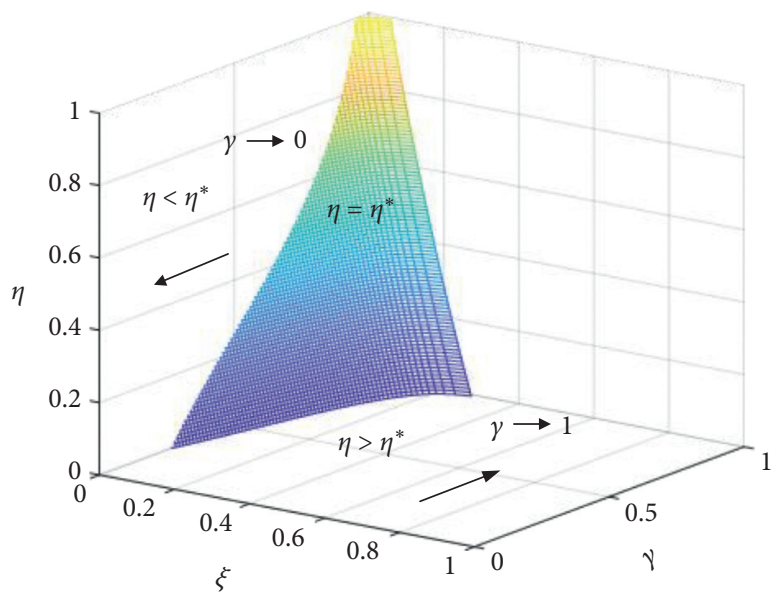

FIGURE 4: Evolutionary phase diagram of consumer's strategy selection.

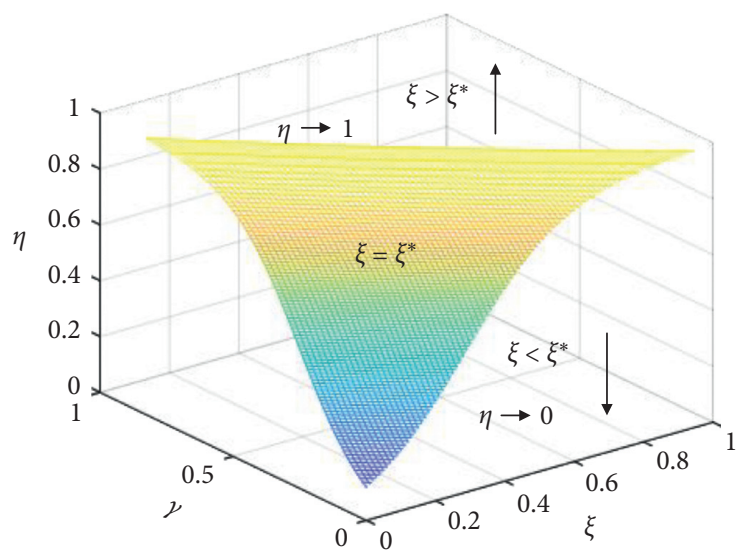

FIGURE 5: Evolutionary phase diagram of central government's strategy selection.

Scenario 1: according to equation (14), if $E_{1 G}-E_{2 G}<0$, $\gamma W+B_{31}-\gamma L_{31}-k S<0$, that is $\xi>\xi^{*}$, then $\left.(\mathrm{d} F(\eta) / \mathrm{d} \eta)\right|_{\eta=0}<0,\left.\quad(\mathrm{~d} F(\eta) / \mathrm{d} \eta)\right|_{\eta=1}>0$. Thus $\eta=0$ is the evolutionary stable point, which represents the globally unique evolutionary stable strategy. It indicates that the central government tends to choose weak supervision as their stable strategy. When the probability of performing food safety risk communication duties by local government is small enough, the central government tends to chooses weak supervision, and weak supervision would be their optimal strategy.

If $E_{1 G}-E_{2 G}<0, \quad \gamma W+B_{31}-\gamma L_{31}-k S>0$, that is, $\xi<\xi^{*}$, then $\left.(\mathrm{d} F(\eta) / \mathrm{d} \eta)\right|_{\eta=0}<0,\left.\quad(\mathrm{~d} F(\eta) / \mathrm{d} \eta)\right|_{\eta=1}>0$. Thus $\eta=0$ is the evolutionary stable point, which represents the globally unique evolutionary stable strategy. It indicates that the central government tends to choose weak supervision as their stable strategy. When the probability of performing food safety risk communication duties by local government is small enough, the central government tends to choose weak supervision. That is to say, weak supervision would be their optimal strategy.
Scenario 2: if $E_{1 G}-E_{2 G}>0, \gamma W+B_{31}-\gamma L_{31}-k S<0$, that is $\xi<\xi^{*}$, then $\left.(\mathrm{d} F(\eta) / \mathrm{d} \eta)\right|_{\eta=0}>0$, $\left.(\mathrm{d} F(\eta) / \mathrm{d} \eta)\right|_{\eta=1}<0$. Thus $\eta=1$ is the evolutionary stable point. It means that the stable point is the globally unique evolutionary stable strategy. It indicates that the central government will tend to choose strong supervision as their stable strategy. When the probability of performing food safety risk communication duties by local government is large enough, the central government tends to choose strong supervision. That is to say, the strong supervision would be their optimal strategy.

If $E_{1 G}-E_{2 G}>0, \gamma W+B_{31}-\gamma L_{31}-k S>0$, that is $\xi>\xi^{*}$, then $\left.(\mathrm{d} F(\eta) / \mathrm{d} \eta)\right|_{\eta=0}>0,\left.(\mathrm{~d} F(\eta) / \mathrm{d} \eta)\right|_{\eta=1}<0$. Thus $\eta=1$ is the evolutionary stable point. It means that the stable point is the globally unique evolutionary stable strategy. It indicates that the central government will choose strong supervision as their stable strategy. When the probability of performing food safety risk communication duties by local government is large enough, the central government will tend to strong supervision. That is to say, the strong supervision would be their optimal strategy. 
4.2.4. Stability Analysis of the Equilibrium Point. In addition, there is an equilibrium solution $E\left(\xi^{*}, \gamma^{*}, \eta^{*}\right)$ that satisfy the following equation:

$$
\left\{\begin{array}{l}
\gamma \eta\left(R-B_{11}\right)+\eta\left(B_{11}+k S-\pi k S\right)+\gamma\left(B_{11}+L_{11}+F\right)+\pi k S+C_{12}-C_{11}-F=0, \\
\xi\left(D_{21}-D_{22}-D_{23}+C_{22}+L_{21}\right)+\xi \eta B_{22}+\eta B_{21}+D_{23}-C_{21}=0, \\
\xi \gamma\left(W-L_{31}\right)+\xi\left(B_{31}-k S\right)+\gamma L_{31}+k S+C_{32}-C_{31}=0 .
\end{array}\right.
$$

Based on the Lyapunov stability theory, the equilibrium point is asymptotically stable when all eigenvalues of the Jacobian matrix are negative. Otherwise, it is unstable. The Jacobian matrix of the system is as follows:

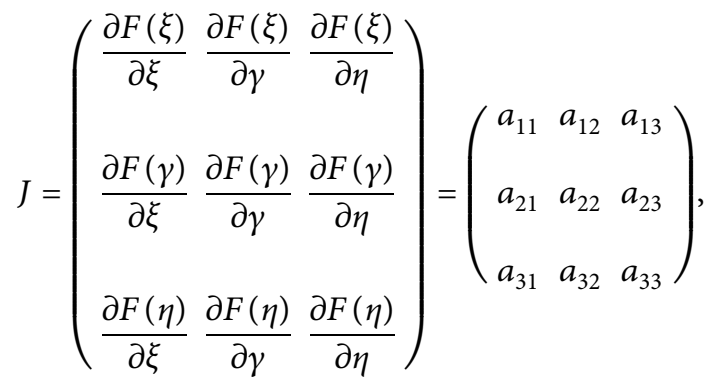

in which $a_{11}=(1-2 \xi)\left(E_{1 L}-E_{2 L}\right), a_{12}=\xi(1-\xi)[\eta(R-$ $\left.\left.B_{11}\right)+B_{11}+L_{11}+F\right]$,

$$
\begin{aligned}
& a_{13}=\xi(1-\xi)\left[\gamma\left(R-B_{11}\right)+B_{11}+k S-\pi k S\right], \\
& a_{21}=\gamma(1-\gamma)\left(D_{21}-D_{22}-D_{23}+C_{22}+L_{21}+\eta B_{22}\right), \\
& a_{22}=(1-2 \gamma)\left(E_{1 C}-E_{2 C}\right), \\
& a_{23}=\gamma(1-\gamma)\left(B_{21}+\xi B_{22}\right), \\
& a_{31}=\eta(1-\eta)\left(\gamma W+B_{31}-\gamma L_{31}-k S\right), \\
& a_{32}=\eta(1-\eta)\left(\xi W-\xi L_{31}+L_{31}\right), \\
& a_{33}=(1-2 \eta)\left(E_{1 G}-E_{2 G}\right) .
\end{aligned}
$$

According to equation (16), we obtained eight evolutionary stable points for local government, consumer, and central government and their conditions.

The equilibrium point $E_{1}(0,0,0)$ is analyzed. The Jacobian matrix at this point is as follows:

$$
J_{1}=\left(\begin{array}{lll}
b & 0 & 0 \\
0 & c & 0 \\
0 & 0 & d
\end{array}\right)
$$

in which $b=\pi k S+C_{12}-C_{11}-F, \quad c=D_{23}-C_{21}$, and $d=k S+C_{32}-C_{31}$.

The concrete values of each element in Table 3 can be written as follows:

$$
\begin{aligned}
& e=B_{11}+k S+C_{12}-C_{11}-F, \\
& f=B_{21}+D_{23}-C_{21}, \\
& g=\pi k S+B_{11}+L_{11}+C_{12}-C_{11}, \\
& a=k S+C_{32}-C_{31}+L_{31}, \\
& h=D_{21}-C_{21}-D_{22}+C_{22}+L_{21}, \\
& i=B_{31}-C_{31}+C_{32}, \\
& j=W+B_{31}-C_{31}+C_{32}, \\
& m=D_{21}-C_{21}-D_{22}+C_{22}+L_{21}+B_{21}+B_{22}, \\
& n=R+k S+B_{11}+L_{11}+C_{12}-C_{11} .
\end{aligned}
$$

We get the eigenvalues $\lambda_{1}=b, \lambda_{2}=c, \lambda_{3}=d$. The asymptotic stability condition is $b<0, c<0, d<0$. Similarly, we can get the eigenvalues and asymptotic stability conditions of $E_{2}, E_{3}, E_{4}, E_{5}, E_{6}, E_{7}$, and $E_{8}$, as shown in Table 3. Furthermore, there are four equilibrium points of single-species adopting pure strategies: $E_{9}(0,(d /(d-a)),(c /(c-f))$, $E_{10}\left((d /(d-i)), 0,(b /(b-e)), E_{11}((c / c-h),(b /(b-g)), 0)\right.$ and $E_{12}(1,(i /(i-j))),(h /(h-m))$.

When $\xi=0,0<\gamma<1,0<\eta<1$, if $F(\gamma)=0, F(\eta)=0$, that is $\eta=\left(\left(C_{21}-D_{23}\right) / B_{21}\right), \gamma=\left(\left(C_{31}-C_{32}-k S\right) / L_{31}\right)$, the conditions for the existence of $E_{9}$ is $0<\left(\left(C_{31}-C_{32}-k S\right) / L_{31}\right)<1,0<\left(\left(C_{21}-D_{23}\right) / B_{21}\right)<1$. At this point, the eigenvalues of the Jacobian matrix are shown as follows:

$$
\begin{aligned}
& A_{1}=\frac{d c(n-e)+c e(b-a)+f(a b-d g)}{(a-d)(f-c)}, \\
& A_{2}=\sqrt{\frac{a c d f}{(a-d)(f-c)}} .
\end{aligned}
$$

When $\gamma=0,0<\xi<1,0<\eta<1$, if $F(\xi)=0, F(\eta)=0$, that is $\xi=\left(C_{31}-C_{32}-k S / B_{31}-k S\right), \eta=\left(\left(C_{11}-C_{12}+F-\right.\right.$ $\left.\pi k S) /\left(B_{11}+k S-\pi k S\right)\right)$, the conditions for the existence of $E_{10}$ are $0<\left(\left(C_{31}-C_{32}-k S\right) /\left(B_{31}-k S\right)\right)<1,0<\left(\left(C_{11}-C_{12}\right.\right.$ $\left.+F-\pi k S) /\left(B_{11}+k S-\pi k S\right)\right)<1$. At this point, the eigenvalues of the Jacobian show the following: 
TABLE 3: Eigenvalues and asymptotic stability conditions of the Jacobian matrix.

\begin{tabular}{|c|c|c|c|c|}
\hline Equilibrium points & Eigenvalues $\lambda_{1}$ & Eigenvalues $\lambda_{2}$ & Eigenvalues $\lambda_{3}$ & Asymptotic stability condition \\
\hline$E_{1}(0,0,0)$ & $b$ & $c$ & $d$ & $b<0, c<0, d<0$ \\
\hline$E_{2}(0,0,1)$ & $e$ & $f$ & $-d$ & $e<0, f<0, d>0$ \\
\hline$E_{3}(0,1,0)$ & $g$ & $-c$ & $a$ & $g<0, c>0, a<0$ \\
\hline$E_{4}(1,0,0)$ & $-b$ & $h$ & $i$ & $b>0, h<0, i<0$ \\
\hline$E_{5}(1,1,0)$ & $-g$ & $-h$ & $j$ & $g>0, h>0, j<0$ \\
\hline$E_{6}(1,0,1)$ & $-e$ & $m$ & $-i$ & $e>0, m<0, i>0$ \\
\hline$E_{7}(0,1,1)$ & $n$ & $-f$ & $-a$ & $n<0, f>0, a>0$ \\
\hline$E_{8}(1,1,1)$ & $-n$ & $-m$ & $-j$ & $n>0, m>0, j>0$ \\
\hline$E_{9}(0,(d /(d-a)),(c /(c-f))$ & $A_{1}$ & $A_{2}$ & $-A_{2}$ & Saddle point \\
\hline$E_{10}((d /(d-i))), 0,(b /(b-e))$ & $A_{3}$ & $A_{4}$ & $-A_{4}$ & Saddle point \\
\hline$E_{11}((c / c-h),(b /(b-g)), 0)$ & $A_{5}$ & $A_{6}$ & $-A_{6}$ & Saddle point \\
\hline$E_{12}(1,(i /(i-j)),(h /(h-m))$ & $A_{7}$ & $A_{8}$ & $-A_{8}$ & Saddle point \\
\hline
\end{tabular}

$$
\begin{aligned}
& A_{3}=\frac{b f i-d e h+b d m+c e i}{(b-e)(d-i)}, \\
& A_{4}=\sqrt{\frac{b d e i}{(b-e)(d-i)}} .
\end{aligned}
$$

When $\eta=0,0<\xi<1,0<\gamma<1$, if $F(\xi)=0, F(\gamma)=0$, that is $\xi=\left(\left(C_{21}-D_{23}\right) /\left(D_{21}-D_{22}-D_{23}+C_{22}+L_{21}\right)\right)$, $\gamma=\left(\left(C_{11}-C_{12}+F-\pi k S\right) /\left(B_{11}+L_{11}+F\right)\right)$, the conditions for the existence of $E_{11}$ is $0<\left(\left(C_{21}-D_{23}\right) /\left(D_{21}-D_{22}-D_{23}\right.\right.$ $\left.\left.+C_{22}+L_{21}\right)\right)<1, \quad 0<\left(\left(C_{11}-C_{12}+F-\pi k S\right) /\left(B_{11}+L_{11}+\right.\right.$ $F))<1$. At this point, the eigenvalues of the Jacobian matrix show the following: $A_{5}=((b c j-c g i-a b h+d g h) /(b-$ g) $(c-h)), A_{6}=\sqrt{(b c g h /(b-g)(c-h))}$.

When $\xi=1,0<\gamma<1,0<\eta<1$, if $F(\gamma)=0, F(\eta)=0$, that is $\eta=\left(\left(D_{22}-C_{22}+C_{21}-D_{21}-L_{21}\right) /\left(B_{21}+B_{22}\right)\right), \gamma=$ $\left(\left(C_{31}-C_{32}-B_{31}\right) / W\right)$, the conditions for the existence of $E_{12}$ are $0<\left(\left(D_{22}-C_{22}+C_{21}-D_{21}-L_{21}\right) /\left(B_{21}+B_{22}\right)\right)<1$, $0<\left(\left(C_{31}-C_{32}-B_{31}\right) / W\right)<1$. At this point, the eigenvalues of the Jacobian matrix are as follows: $A_{7}=(e h j+g i m-$ bjm-hin/ $(i-j)(h-m)), A_{8}=\sqrt{(h i j m /(i-j)(h-m))}$.

\section{Numerical Simulation}

According to the player's stable strategies above, to verify the evolutionary game model and analysis results, the impact of relevant variables has been analyzed by numerical simulation. This study uses Matlab2018a to simulate the evolution process of strategy selection by the tripartite. The dynamic equations (3), (6), (9) can discretize the asymptotically stable trajectory of the tripartite evolutionary game. Let $\Delta t$ be a time step. The tripartite dynamic system could be formulated as follows:

$$
\left\{\begin{array}{l}
\frac{\mathrm{d} \xi(t)}{\mathrm{d} t} \approx \frac{\xi(t+\Delta t)-\xi(t)}{\Delta t}=\xi(1-\xi)\left[\gamma \eta P_{1}+\eta P_{2}+\gamma P_{3}+b\right], \\
\frac{\mathrm{d} \gamma(t)}{\mathrm{d} t} \approx \frac{\gamma(t+\Delta t)-\gamma(t)}{\Delta t}=\gamma(1-\gamma)\left[\xi P_{4}+\eta P_{5}+\eta \xi P_{6}+c\right], \\
\frac{\mathrm{d} \eta(t)}{\mathrm{d} t} \approx \frac{\eta(t+\Delta t)-\eta(t)}{\Delta t}=\eta(1-\eta)\left[\xi \gamma P_{7}+\xi P_{8}+\gamma P_{9}+d\right],
\end{array}\right.
$$

in which $P_{1}=R-B_{11}, \quad P_{2}=B_{11}+k S-\pi k S, \quad P_{3}=B_{11}+$ $L_{11}+F, P_{5}=B_{21}, \quad P_{6}=B_{22}, \quad P_{4}=D_{21}-D_{22}-D_{23}+C_{22}+$ $L_{21}, P_{7}=W-L_{31}, P_{8}=B_{31}-k S$, and $P_{9}=L_{31}$

Setting the time step $\Delta t=1$, the upper dynamic system becomes as follows:

$$
\left\{\begin{array}{l}
\xi(t+1)=\xi(t)[2-\xi(t)]\left[\gamma(t) \eta(t) P_{1}+\eta(t) P_{2}+\gamma(t) P_{3}+b\right] \\
\gamma(t+1)=\gamma(t)[2-\gamma(t)]\left[\xi(t) P_{4}+\eta(t) P_{5}+\eta(t) \xi(t) P_{6}+c\right] \\
\eta(t+1)=\eta(t)[2-\eta(t)]\left[\xi(t) \gamma(t) P_{7}+\xi(t) P_{8}+\gamma(t) P_{9}+d\right] .
\end{array}\right.
$$

This is a recursive formula for the probability of the local government, consumer, and the central government choose "perform food safety risk communication duties," "proactive communication," and "strong supervision" in the evolutionary process. The parameters required to be assigned in the model are $R, \pi, k, S, F, W, L_{11}, D_{21}, D_{22}, D_{23}, C_{22}, L_{21}, B_{11}$, $B_{21}, B_{22}, L_{31}, B_{31}, C_{12}, C_{11}, C_{21}, C_{31}, C_{32}$.

According to equation (16), we have obtained eight evolutionary stable points of regulators and consumers in the evolutionary game model under constraint conditions (see Table 3). Among them, $E_{1}$ (not perform food safety risk communication duties, reactive communication, weak supervision), $E_{2}$ (not perform food safety risk communication duties, reactive communication, strong supervision) are the worst outcome. Therefore, we should ignore the conditions of these stable points from a rational point of view. $E_{8}$ (perform food safety risk communication duties, proactive communication, weak supervision) is the ideal outcome. However, it is difficult to achieve the ideal stable point result in actual risk communication. The reality is often more complicated. Meanwhile, $E_{5}(1,1,0), E_{7}(0,1,1)$, and $E_{6}(1,0$, 1 ) are the relatively ideal outcomes (see Table 4 ). Other stable points are discussed below.

Scenario 1: to perform food risk communication duties, proactive communication, and strong supervision.

The stable equilibrium point $E_{8}(1,1,1)$ expected by the research, the parameter values should satisfy the following conditions: $R+k S+B_{11}+L_{11}+C_{12}-C_{11}>0$, $W+B_{31}-C_{31}+C_{32}>0$,

$D_{21}-C_{21}-D_{22}+C_{22}+L_{21}+B_{21}+B_{22}>0$. We assume the scenarios for the initial probability of performing food risk communication duties, proactive communication, and strong supervision. 
TABLE 4: Strategy space of the tripartite game model.

\begin{tabular}{lcc}
\hline Equilibrium point & Strategy space & Policy effectiveness \\
\hline$E_{8}(1,1,1)$ & Perform risk communication duties, proactive communication, strong supervision & Most ideal \\
$E_{5}(1,1,0)$ & Perform risk communication duties, proactive communication, weak supervision & Ideal \\
$E_{7}(0,1,1)$ & Not to perform risk communication duties, proactive communication, strong supervision & Medium \\
$E_{4}(1,0,0)$ & Perform risk communication duties, reactive communication, weak supervision & \\
$E_{3}(0,1,0)$ & Not to perform risk communication duties, proactive communication, weak supervision & \\
$E_{6}(1,0.1)$ & Perform risk communication duties, reactive communication, strong supervision & Least ideal \\
\hline
\end{tabular}

Let the parameters be $R=20, \pi=0.6, k=0.2, S=6$, $F=10, W=13, L_{11}=9, D_{21}=29, D_{22}=18, D_{23}=16$, $C_{22}=8, L_{21}=11, B_{11}=3, B_{21}=4, B_{22}=5, L_{31}=7$, $B_{31}=17, C_{12}=5, C_{11}=6, C_{21}=10, C_{31}=9, C_{32}=4$. According to the simulation results (see Figure 6) show that although the initial probability of three players' strategy choice has a significant effect on the evolution path, the evolution path of evolutionary stable points formed by the tripartite is the same over time. When satisfying the above conditions, the equilibrium point is $E_{8}(1,1,1)$. Over time, local government tends to perform food safety risk communication duties, the consumer tends to choose proactive communication on food risk, and the central government tends to choose strong supervision strategy. Moreover, the reward from the central government has a significant impact on the local government, which can be seen from Figure 6 that increases the proportion of performing food safety risk communication duties by local government. Similarly, the return of consumers has a significant positive impact on food risk communication attitude activity of consumers. The long-term social welfare increase would improve the probability of strong supervision of the central government. Thus, the stable equilibrium point $E_{8}(1,1,1)$ is an ideal situation.

Scenario 2: to perform food risk communication duties, proactive communication, and weak supervision.

The stable equilibrium point $E_{5}(1,1,0)$ is expected by the research, the parameter values should satisfy the following conditions: $\pi k S+B_{11}+L_{11}+C_{12}-C_{11}>0$, $W+B_{31}-C_{31}+C_{32}<0$,

$D_{21}-C_{21}-D_{22}+C_{22}+L_{21}>0$. We assume the scenarios for the initial probability of perform food risk communication duties, proactive communication, and weak supervision.

Let the parameters be $R=20, \pi=0.6, k=0.2, S=6$, $F=10, W=3, L_{11}=9, D_{21}=29, D_{22}=18, D_{23}=16$, $C_{22}=8, L_{21}=11, B_{11}=3, B_{21}=4, B_{22}=5, L_{31}=7$, $B_{31}=4, C_{12}=5, C_{11}=6, C_{21}=10, C_{31}=10, C_{32}=2$. The simulation results (see Figure 7 ) show that the parameters have a significant impact on the evolutionary path of strategies apart from the initial probabilities of strategy choices. When satisfying the above conditions, the equilibrium point is $E_{5}(1,1,0)$. Over time, the local government chooses to perform food safety risk communication duties, the consumer chooses proactive communication, and the central government chooses weak supervision. The results

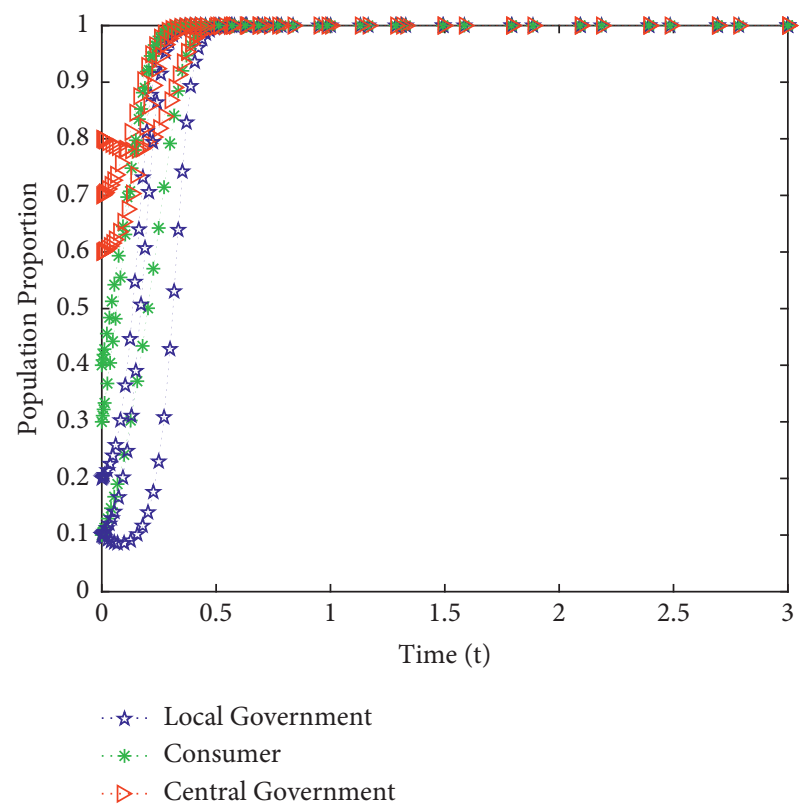

Figure 6: Evolution path of strategies chosen by three players with the stability point $\mathrm{E}_{8}$.

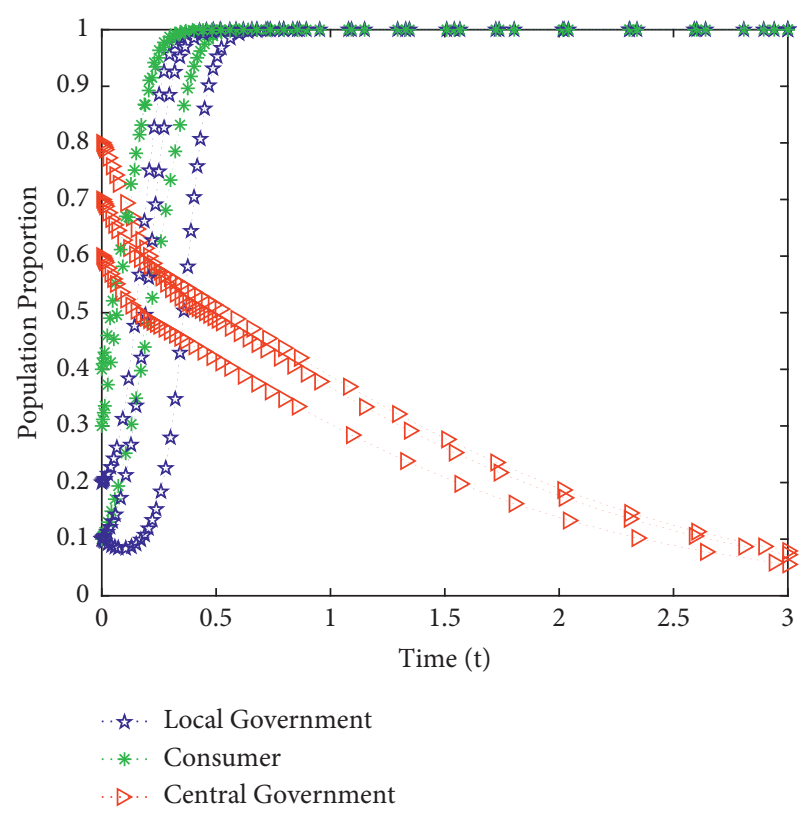

FIgURE 7: Evolution path of strategies chosen by three players with the stability point $\mathrm{E}_{5}$. 
indicate that the decrease in social welfare and credibility to central government will have a significant impact on the evolution path of strategy. The central government will choose weak supervision. At this time, the stable equilibrium point $E_{5}(1,1,0)$ is an ideal point.

Scenario 3: not to perform food risk communication duties, proactive communication, and strong supervision.

The stable equilibrium point $E_{7}(0,1,1)$ is expected by the research, the parameter values should satisfy the following conditions: $R+k S+B_{11}+L_{11}+C_{12}-C_{11}<$ $0, \quad B_{21}+D_{23}-C_{21}>0, \quad k S+C_{32}-C_{31}+L_{31}>0$. We assume the scenarios for the initial probability of not performing food risk communication duties, proactive communication, and strong supervision.

Let the parameters be $R=3, \pi=0.6, k=0.2, S=6$, $F=10, W=3, L_{11}=4, D_{21}=29, D_{22}=18, D_{23}=16$, $C_{22}=8, L_{21}=11, B_{11}=3, B_{21}=4, B_{22}=5, L_{31}=7$, $B_{31}=4, \quad C_{12}=5, \quad C_{11}=20, \quad C_{21}=10, \quad C_{31}=10$, $C_{32}=12$. The simulation results (see Figure 8) show that the parameters have a significant impact on the evolutionary path of strategies apart from the initial probabilities of strategy choices. When satisfying the above conditions, the equilibrium point is $E_{7}(0,1,1)$. Over time, the local government chooses not to perform food safety risk communication duties, the consumer chooses proactive communication, and the central government chooses strong supervision. The results indicate that increased cost on performing food risk communication duties and implementing weak supervision to regulators will significantly impact the evolution path of strategy. Finally, the system evolves towards the stable equilibrium point $E_{7}(0,1,1)$.

Scenario 4: performing food risk communication duties, reactive communication, and weak supervision.

The stable equilibrium point $E_{4}(1,0,0)$ is expected by the research, the parameter values should satisfy the following conditions: $\pi k S+C_{12}-C_{11}-F>0$, $D_{21}-C_{21}-D_{22}+C_{22}+L_{21}<0, \quad B_{31}-C_{31}+C_{32}<0$. We assume the scenarios for the initial probability of performing food risk communication duties, reactive communication, and weak supervision.

Let the parameters be $R=3, \pi=0.6, k=0.2, S=6$, $F=3, W=3, L_{11}=4, D_{21}=20, D_{22}=18, D_{23}=16$, $C_{22}=3, L_{21}=4, B_{11}=3, B_{21}=4, B_{22}=3, L_{31}=7$, $B_{31}=4, C_{12}=8, C_{11}=2, C_{21}=16, C_{31}=10, C_{32}=2$. The simulation results (see Figure 9) show that as the iteration process proceeds, the proportion of consumers adopting proactive communication and the proportion of the central government implementing strong supervision decrease while the proportion of local government performing food safety risk communication duties increases. As long as the cost of performing food risk communication duties is lower than the reward from the central government and the potential loss decreases with performing risk communication duties, the local government will perform

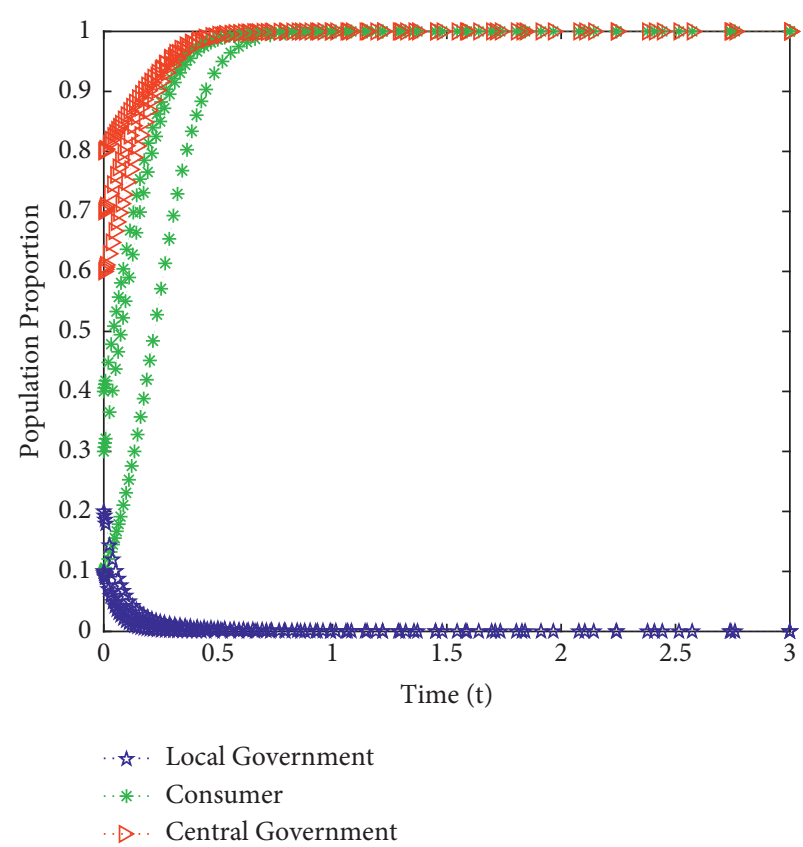

FIGURE 8: Evolution path of strategies chosen by three players with the stability point $\mathrm{E}_{7}$.

the food safety risk communication duties. It indicates that the cost parameters have a significant impact on the evolutionary path of strategies apart from the initial probabilities of strategy choices. When satisfying the above conditions, the equilibrium point is $E_{4}(1,0,0)$ over time, the local government chooses to perform food safety risk communication duties, while the consumer chooses reactive communication, and the central government chooses weak supervision. Finally, the system evolves towards the stable equilibrium point $E_{4}(1,0,0)$.

Scenario 5: not to perform food risk communication duties, proactive communication, and weak supervision.

The stable equilibrium point $E_{3}(0,1,0)$ is expected by the research, the parameter values should satisfy the following conditions: $\pi k S+B_{11}+L_{11}+C_{12}-C_{11}<0$, $D_{23}-C_{21}>0, k S+C_{32}-C_{31}+L_{31}<0$. We assume the scenarios for the initial probability of not performing food risk communication duties, proactive communication, and weak supervision.

Let the parameters be $R=3, \pi=0.6, k=0.2, S=6$, $F=3, W=3, L_{11}=4, D_{21}=20, D_{22}=18, D_{23}=16$, $C_{22}=3, L_{21}=4, B_{11}=3, B_{21}=4, B_{22}=5, L_{31}=5$, $B_{31}=4, C_{12}=5, C_{11}=14, C_{21}=7, C_{31}=10, C_{32}=2$. The simulation results (see Figure 10) show that, as the iteration process proceeds, the proportion of local government performs food safety risk communication duties. The proportion of the central government implementing strong supervision decreases while the proportion of consumers adopting proactive communication increases. As long as the cost of consumers implementing proactive communication on food risk is 


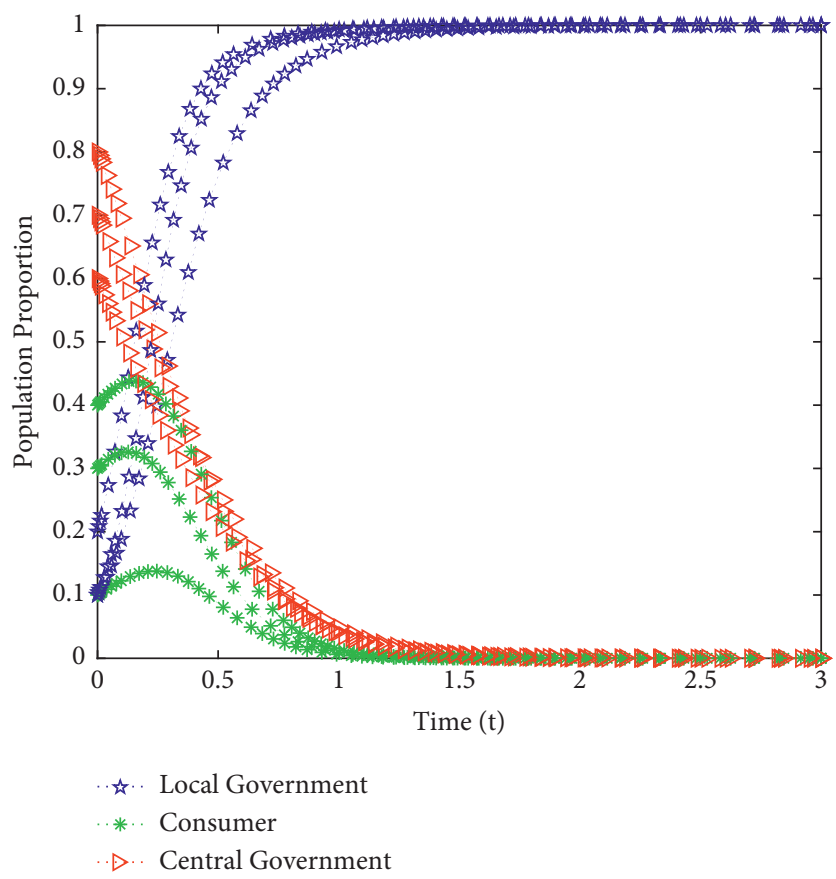

FIgURE 9: Evolution path of strategies chosen by three players with the stability point $\mathrm{E}_{4}$.

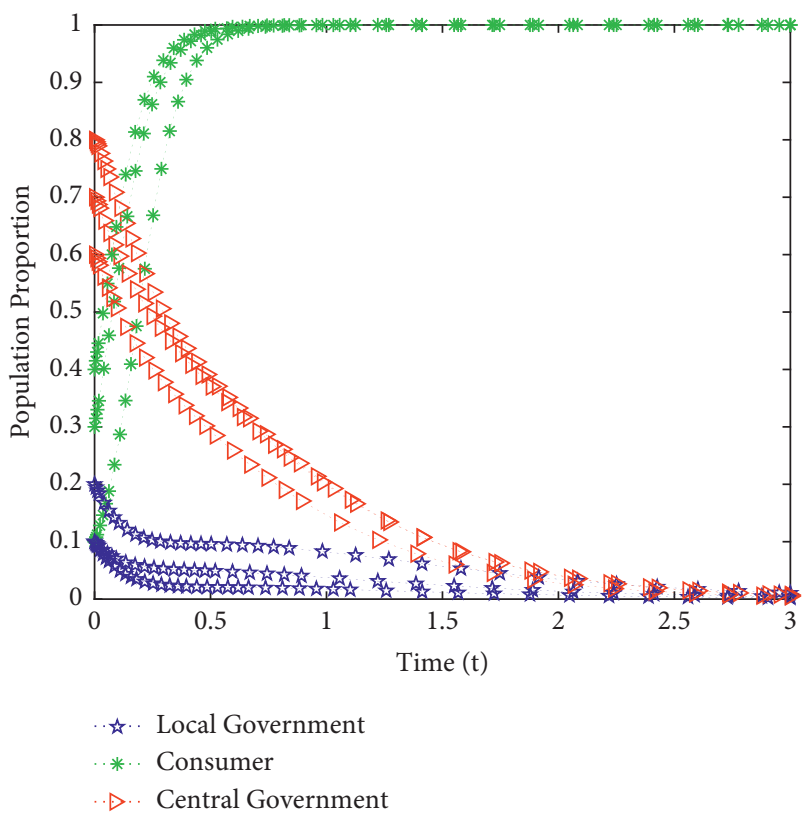

FIGURE 10: Evolution path of strategies chosen by three players with the stability point $\mathrm{E}_{3}$.

lower than the return, the consumer will choose proactive communication on food risk. Therefore, it can be seen from the evolution result that the consumer's risk communication initiative is affected by the benefit obtained from communication. When satisfying the above conditions, the equilibrium point is $E_{3}(0,1,0)$. Over time, the local government fails to perform food safety risk communication duties, while the consumer chooses proactive communication, and the central government chooses weak supervision. Finally, the system evolves towards the stable equilibrium point $E_{3}$ $(0,1,0)$.

Scenario 6: performing food risk communication duties, reactive communication, and strong supervision.

The stable equilibrium point $E_{6}(1,0,1)$ expected by the research, the parameter values should satisfy the following conditions: $B_{11}+k S+C_{12}-C_{11}-F>0, B_{31}-C_{31}+C_{32}>0$, $D_{21}-C_{21}-D_{22}+C_{22}+L_{21}+B_{21}+B_{22}<0$. We assume the scenarios for the initial probability of performing food risk communication duties, reactive communication, and strong supervision.

Let the parameters be $R=3, \pi=0.6, k=0.2, S=6, F=3$, $W=3, L_{11}=4, D_{21}=16, D_{22}=18, D_{23}=16, C_{22}=3$, $L_{21}=2, B_{11}=3, B_{21}=2, B_{22}=3, L_{31}=5, B_{31}=4, C_{12}=5$, $C_{11}=2, C_{21}=13, C_{31}=10, C_{32}=8$. The simulation results (see Figure 11) show that as the iteration process proceeds, the proportion of local government is performing food safety risk communication duties. The proportion of the central government implementing strong supervision increases while the proportion of consumers' proactive communication decreases. As long as the return of consumers implementing proactive communication on food risk decreases, the consumer will choose reactive communication on food risk. Therefore, the evolution result indicates that the consumer's risk communication initiative is affected by the benefit obtained from communication. When satisfying the above conditions, the equilibrium point is $E_{6}(1,0,1)$. Over time, the local government chooses to perform food safety risk communication duties, while the consumer chooses reactive communication, and the central government chooses strong supervision. Finally, the system evolves towards the stable equilibrium point $E_{6}(1,0,1)$. 


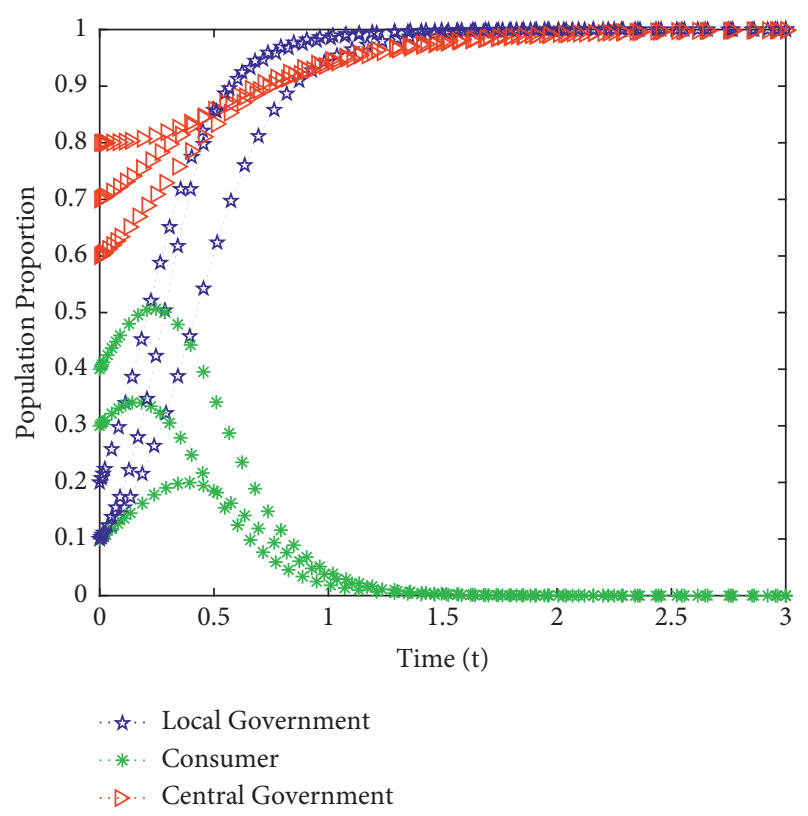

FIGURE 11: Evolution path of strategies chosen by three players with the stability point $\mathrm{E}_{6}$.

\section{Conclusion and Enlightenment}

This research provides a tripartite evolutionary game model involving regulators (local government and central government) and consumers in China for exploring the food safety risk communication system. Equilibrium points of the game and the evolutionarily stable strategy of each agent have been obtained by solving the replicator dynamic equation. Through the evolutionary phase diagram of each agent and numerical simulation, we analyzed the globally unique evolutionary stable strategy of the game model. The stability analysis at the equilibrium points and considering the actual situation conclude that the ideal evolutionary stable strategy is to "perform food safety risk communication duties, proactive communication, strong supervision." This strategy provides a guarantee for the governance of food safety and the prevention of consumer exposure to food safety issues.

This study shows food safety risk communication between local government, central government, and consumers. It will help to raise the awareness of the public on food risk issues, effectively push the government to pay attention to food safety risk communication by properly adjusting the intensity of supervision, and encourage the public to proactive communication on food risk issues. Proactive and reactive communication are the main food safety risk types of communication by consumers. However, due to time cost and limited knowledge, consumers are inert in food safety risk communication, making reactive communication for food risk between consumer and regulator the common state in China. Therefore, concise and easy-tounderstand language is very important for low-literate audience to understand food safety risk knowledge. In addition, food risk communication is needed to establish trust between the public and regulator through ongoing actions, listening, and coordination skill. The local government and central government could take measures to encourage consumers to change their risk communication concept and search food information, interact with information publishers, increase their understanding of food safety risks, and reduce the probability of food safety accidents.

Furthermore, there is enlightening guidance for government and consumers to reach the ideal evolutionary strategy. Firstly, the government should accept and involve the public as a legitimate partner in food risk communication. Listening to the public's specific concerns is a vital facet to improve understanding of public values. A better understanding of the public would allow the regulator to select the appropriate means and outlets to connect with specific intended receivers. On the other hand, the government should educate the public about food risks, risk analysis, and risk management to alleviate them. Secondly, the regulator must be honest, frank, and open in food risk communication. Especially for the public in China, social trust in the government and institutions has a vital impact on perceived risk, thus on food risk communication. Effective risk communication must maintain the trust situation of the target audience because people only accept or interact with the messages from what they trust. Better strategies are contributing to increase mutual trust and credibility between the authorities and the public through transparency food information. Thirdly, the food information communicated must meet the needs of the consumer. Regulators should identify vulnerable populations, pretest messages to focus groups, and deliver key factors of food risk.

\section{Data Availability}

The data used to support the findings of this study have not been made available because raw data are hard to obtain. So, this study uses hypothetical data for simulation rather than real data.

\section{Conflicts of Interest}

The authors declare that they have no conflicts of interest.

\section{Acknowledgments}

This work was supported by the National Natural Science Foundation of China (no. 71633002), the International Training Program for Outstanding Young Scientists in Universities in Guangdong Province (no. 2020YQGP_BS002), and the Young Innovation Talents Program at the University of Guangdong Province (no. 2018WQNCX294).

\section{References}

[1] L. J. Frewer and A. R. Fischer, "Risk analysis: risk communication," Encyclopedia of Food Safety, vol. 1, pp. 116-121, 2014.

[2] D. A. Powell, "Food safety and the consumer-perils of poor risk communication," Canadian Journal of Animal Science, vol. 80, no. 3, pp. 393-404, 2000. 
[3] European Food Safety Authority, When Food Is Cooking up a Storm-Proven Recipes for Risk Communications, European Food Safety Authority, Parma, Italy, 2012.

[4] Á. Regan, M. Raats, L. C. Shan, P. G. Wall, and Á. McConnon, "Risk communication and social media during food safety crises: a study of stakeholders' opinions in Ireland," Journal of Risk Research, vol. 19, no. 1, pp. 1-14, 2014.

[5] Food Safety Commission, Risk Communication \& Food Additives 2020, Food Safety Commission, Tokyo, Japan, 2020.

[6] J. M. Antle, "Benefits and costs of food safety regulation," Food Policy, vol. 24, no. 6, pp. 605-623, 1999.

[7] A. Pinto, G. Mascarello, N. Parise, S. Bonaldo, S. Crovato, and L. Ravarotto, "Italian consumers' attitudes towards food risks: self-protective and non-self-protective profiles for effective risk communication," Journal of Risk Research, vol. 20, no. 3, 2015.

[8] S. Charlebois and A. Summan, "A risk communication model for food regulatory agencies in modern society," Trends in Food Science \& Technology, vol. 45, no. 1, 2015.

[9] S. Yang, M. G. Leff, D. McTague et al., "Multistate surveillance for food-handling, preparation, and consumption behaviors associated with foodborne diseases: 1995 and 1996 BRFSS food-safety questions. MMWR. CDC surveillance summaries: morbidity and mortality weekly report," CDC Surveillance Summaries/Centers for Disease Control, vol. 47, no. 4, pp. 33-57, 1998.

[10] $\AA$. Boholm, "Lessons of success and failure: practicing risk communication at government agencies," Safety Science, vol. 118, pp. 158-167, 2019.

[11] A. Al-Sakkaf, "Campylobacteriosis in New Zealand: a new twist to the tale? Part two (the consumer and the regulator)," Food Control, vol. 33, no. 2, pp. 562-566, 2013.

[12] W. Annabelle, S. B. Meyer, T. Webb et al., "How food regulators communicate with consumers about food safety," British Food Journal, vol. 117, no. 8, pp. 2129-2142, 2015.

[13] Z. Liu, A. N. Mutukumira, and H. Chen, "Food safety governance in China: from supervision to coregulation," Food Science \& Nutrition, vol. 7, no. 1, pp. 4127-4139, 2019.

[14] X. Zhu, I. Y. Huang, and L. Manning, "The role of media reporting in food safety governance in China: a dairy case study," Food Control, vol. 96, pp. 165-179, 2019.

[15] Y.-n. Wu and J.-s. Chen, "Food safety monitoring and surveillance in China: past, present and future," Food Control, vol. 90, pp. 429-439, 2018.

[16] Y. Kang, "Food safety governance in China: change and continuity," Food Control, vol. 106, Article ID 106752, 2019.

[17] M. Chu and J. Wang, "Central-local collaboration in regulating food safety in China," Public Money \& Management, vol. 38 , no. 6, 2018.

[18] C. May, "Horses for courses: China's accommodative approach to food standard-setting in response to the internationalization of regulation," Regulation \& Governance, vol. 14, no. 3, pp. 1-17, 2020.

[19] S. Crovato, A. Pinto, P. Giardullo, G. Mascarello, F. Neresini, and L. Ravarotto, "Food safety and young consumers: testing a serious game as a risk communication tool," Food Control, vol. 62, pp. 134-141, 2016.

[20] C. Hartmann, P. Hübner, and M. Siegrist, "A risk perception gap? Comparing expert, producer and consumer prioritization of food hazard controls," Food and Chemical Toxicology, vol. 116, pp. 100-107, 2018.

[21] J. Hansen, L. Holm, L. Frewer, P. Robinson, and P. Sandøe, "Beyond the knowledge deficit: recent research into lay and expert attitudes to food risks," Appetite, vol. 41, no. 2, pp. 111-121, 2003.

[22] M. Siegrist, C. Keller, H. Kastenholz, S. Frey, and A. Wiek, "Laypeople's and experts' perception of nanotechnology hazards," Risk Analysis, vol. 27, no. 1, pp. 59-69, 2007.

[23] C. Griffith, D. Worsfold, and R. Mitchell, "Food preparation, risk communication and the consumer," Food Control, vol. 9, no. 4, pp. 225-232, 1998.

[24] FAO, Risk Communication Applied to Food Safety, Food and Agriculture Organisation, Rome, Italy, 2016.

[25] J. Li, Y. Ren, W. Ma, and T. Yu, "Food safety and rural parents in China: investigating an effective path for risk communication," Health Communication, vol. 35, no. 14, pp. 1762-1771, 2020.

[26] Y. Song, N. Shen, and D. Liu, "Evolutionary game and intelligent simulation of food safety information disclosure oriented to traceability system," Journal of Intelligent and Fuzzy Systems, vol. 35, no. 3, pp. 2657-2665, 2018.

[27] F. Han and H. Li, "Food safety evolutionary game simulation model based on improved prospect theory," Journal of Interdisciplinary Mathematics, vol. 20, no. 6-7, pp. 1349-1354, 2017.

[28] J. Luo, B. Ma, Y. Zhao, and T. Chen, "Evolution model of health food safety risk based on prospect theory," Journal of healthcare engineering, vol. 2018, Article ID 8769563, 12 pages, 2018.

[29] J. Luo, T. Chen, and J. Pan, "Evolutionary dynamics of health food safety regulatory information disclosure from the perspective of consumer participation," Food Sciences and Nutrition, vol. 7, no. 12, pp. 3958-3968, 2019.

[30] S. Yang, J. Zhuang, A. Wang, and Y. Zhang, "Evolutionary game analysis of Chinese food quality considering effort levels," Complexity, vol. 2019, Article ID 6242745, 13 pages, 2019.

[31] K. Neuwirth, S. Dunwoody, and R. J. Griffin, "Protection motivation and risk communication," Risk Analysis, vol. 20, no. 5, pp. 721-734, 2000.

[32] L. Frewer, "The public and effective risk communication," Toxicology Letters, vol. 149, no. 1-3, pp. 391-397, 2004.

[33] M. S. Erdozain, K. J. Allen, K. A. Morley, and D. A. Powell, "Failures in sprouts-related risk communication," Food Control, vol. 30, no. 2, pp. 649-656, 2013.

[34] Q. Guo, N. Yao, and W. Zhu, "How consumers' perception and information processing affect their acceptance of genetically modified foods in China: a risk communication perspective," Food Research International, vol. 137, Article ID 109518, 2020.

[35] R. E. Lofstedt, "How can we make food risk communication better: where are we and where are we going?," Journal of Risk Research, vol. 9, no. 8, pp. 869-890, 2006.

[36] M. McCarthy and M. Brennan, "Food risk communication: some of the problems and issues faced by communicators on the Island of Ireland (IOI)," Food Policy, vol. 34, no. 6, pp. 549-556, 2009.

[37] $\varnothing$. Ueland, "How to make risk communication influence behavior change," Trends in Food Science \& Technology, vol. 84, pp. 71-73, 2019.

[38] P. G. Wall and J. Chen, "Moving from risk communication to food information communication and consumer engagement," Npj Science of Food, vol. 21, pp. 1-5, 2018.

[39] Y. Zhang and Y. Guizani, Game Theory for Wireless Communications and Networking, CRC Press, Boca Raton, FL, USA, 2011. 
[40] Q. Zhu and T. Başar, "Game-theoretic approach to feedbackdriven multi-stage moving target defense," in Decision and Game Theory for Security, pp. 246-263, Springer, Cham, Switzerland, 2013. 